\title{
The elaboration of numerical simulation error light pulse propagation in a waveguide of circular cross-section
}

\author{
A.A. Degtuarev ${ }^{1}$, A.V. Kukleva ${ }^{1}$

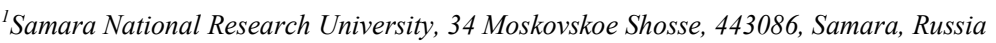

\begin{abstract}
We considered the problem of estimating the error in the solution of the wave equation recorded using infinite series Fourier-Bessel. The algorithm that adjusts the number of elements in a partial sum of infinite series, based on the assessment of the series balance. The application of the algorithm made it possible, without loss of accuracy, to substantially reduce the number of summable elements of the series in the numerical simulation of the light pulse propagation in a circular cross-section.
\end{abstract}

Keywords: wave equation; Fourier-Bessel series; evaluation of the residual series; numerical simulations; pulse of light; computational experiment; redundancy of partial sum components

\section{Introduction}

During the development of an application program for the numerical simulation of a physical process, it is important to investigate the actual error of the method used on special test cases. As test cases typically use such examples that can be resolved by an alternative method with high sufficiently precision, allowing to calculate the error of numerical method [1,2].

This work is devoted to study the error of test value problem for the wave equation describing the propagation process of the light pulse in a waveguide in circular cross section. To elaboration the error estimate, we used remainder of the Fourier-Bessel. To check the quality of the balance assessment in the series we used the technique of computational experiment, which allows determine the degree of redundancy among several elements needed to sum to achieve the necessary precision [3].

In solving problems from numerical simulation propagation of a light pulse in a medium, various mathematical descriptions of the pulse [4-6]. In this paper, we considered two options describe different degrees of smoothness pulse function.

\section{Mathematical model of light pulse propagation in a waveguide of circular cross-section}

To describe the process of light pulse propagation we will consider the following boundary value problem:

$$
\left\{\begin{array}{l}
\frac{\partial^{2} E}{\partial t^{2}}=\frac{c^{2}}{n^{2}}\left(\frac{\partial^{2} E}{\partial r^{2}}+\frac{1}{r} \frac{\partial E}{\partial r}+\frac{\partial^{2} E}{\partial z^{2}}\right), r \in(0 ; R], z \in[0 ; L], t \in[0 ; T] ; \\
\left.E\right|_{t=0}=0, r \in(0 ; R], \quad z \in[0 ; L] ; \\
\left.\frac{\partial E}{\partial t}\right|_{t=0}=0, r \in(0 ; R], \quad z \in[0 ; L] ; \\
\left.E\right|_{z=0}=\psi(r, t), r \in(0 ; R], t \in[0 ; T] ; \\
\left.\frac{\partial E}{\partial z}\right|_{z=L}=0, r \in(0 ; R], t \in[0 ; T] ; \\
\left.E\right|_{r=R}=0, \quad z \in[0 ; L], t \in[0 ; T],
\end{array}\right.
$$

where $E$ is a dielectric field intensity, $c$ is a wave propagation speed in vacuum, $n$ is a refractive index material of the waveguide, $R$ and $L$ is the radius and length of the waveguide, $T$ is the duration of the dissemination process, $\psi(r, t)$ is the function describing the pulse shape.

It is assumed when $r=R$ an ideally conducting shell bound the waveguide, and the medium is not perturbed at the initial instant of time.

Here are the following two variants of kinetic moment:

$$
\psi_{1}(r, t)=\varphi(r) \gamma(t) \sin \omega t, \quad \psi_{2}(r, t)=\varphi(r) \gamma(t) \sin \omega t \sin ^{2} \omega^{*} t
$$

where $\gamma(t)=\left\{\begin{array}{l}1, t \in\left[0 ; t^{*}\right] ; \\ 0, t \in\left(t^{*} ; T_{t}\right],\end{array} \quad \omega=\frac{2 \pi c}{\lambda}, \omega^{*}=\frac{2 \pi c}{\lambda j}, t^{*}\right.$ is the pulse duration at the entrance of the waveguide, $\lambda$ is the length of disturbing wave in vacuum, $j$ a positive integer. $\psi_{1}(r, t)$ a piecewise smooth function at variable $t$, because derivative has function jump in $t=0, t=t^{*}$. Function $\psi_{2}(r, t)$ has the smoothness of a second-order variable $t$. 


\section{Exact solution of boundary value problem}

Application of the separation variables method [5] allows getting solution of boundary-value problem for the wave equation, it can be thought of as infinite series Fourier-Bessel. For example, when describing an impulse function $\psi_{1}(r, t)$ and using $\varphi(r)=J_{0}\left(\lambda_{1} r\right)$ the solution would be:

$$
\begin{aligned}
& E(r, z, t)=J_{0}\left(\lambda_{1} r\right)\left[\sum_{k=0}^{\infty} c_{k} \sin \left(v_{k} z\right) \frac{\omega \sin \left(\omega_{k} t\right)\left(\hat{\omega}^{2}-\omega_{k}^{2}\right)-\omega_{k} \sin (\omega t)\left(\hat{\omega}^{2}-\omega^{2}\right)}{\omega_{k}\left(\omega_{k}^{2}-\omega^{2}\right)}+\sin (\omega t)\right], \text { if } t \in\left[0 ; t^{*}\right] ; \\
& E(r, z, t)=J_{0}\left(\lambda_{1} r\right) \sum_{k=0}^{\infty} \sin \left(v_{k} z\right)\left(a_{1}\left(t^{*}\right) \cos \left(\omega_{k}\left(t-t^{*}\right)\right)+\frac{a_{2}\left(t^{*}\right)}{\omega_{k}} \sin \left(\omega_{k}\left(t-t^{*}\right)\right)\right), \text { if } t \in\left(t^{*} ; T\right] .
\end{aligned}
$$

When writing these formulas, we use the following notation:

$$
\begin{aligned}
& \lambda_{1}=\frac{\mu_{1}}{R}, \quad c_{k}=\frac{4}{\pi(2 k+1)}, \quad v_{k}=\frac{\pi(2 k+1)}{2 L}, \quad \omega_{k}=\frac{c}{n} \sqrt{v_{k}^{2}+\lambda_{1}^{2},} \quad \hat{\omega}=\frac{c}{n} \lambda_{1}, \\
& a_{1}(t)=c_{k}\left[\frac{\omega \sin \left(\omega_{k} t\right)\left(\hat{\omega}^{2}-\omega_{k}^{2}\right)-\omega_{k} \sin (\omega t)\left(\hat{\omega}^{2}-\omega^{2}\right)}{\omega_{k}\left(\omega_{k}^{2}-\omega^{2}\right)}+\sin (\omega t)\right],
\end{aligned}
$$$$
a_{2}(t)=c_{k} \omega\left[\frac{\cos \left(\omega_{k} t\right)\left(\hat{\omega}^{2}-\omega_{k}^{2}\right)-\cos (\omega t)\left(\hat{\omega}^{2}-\omega^{2}\right)}{\left(\omega_{k}^{2}-\omega^{2}\right)}+\cos (\omega t)\right] \text {. }
$$

Graph of the cross section of a pulse by a plane $r=1 \mu m$ in the process of its propagation in the waveguide has shown in fig.1.

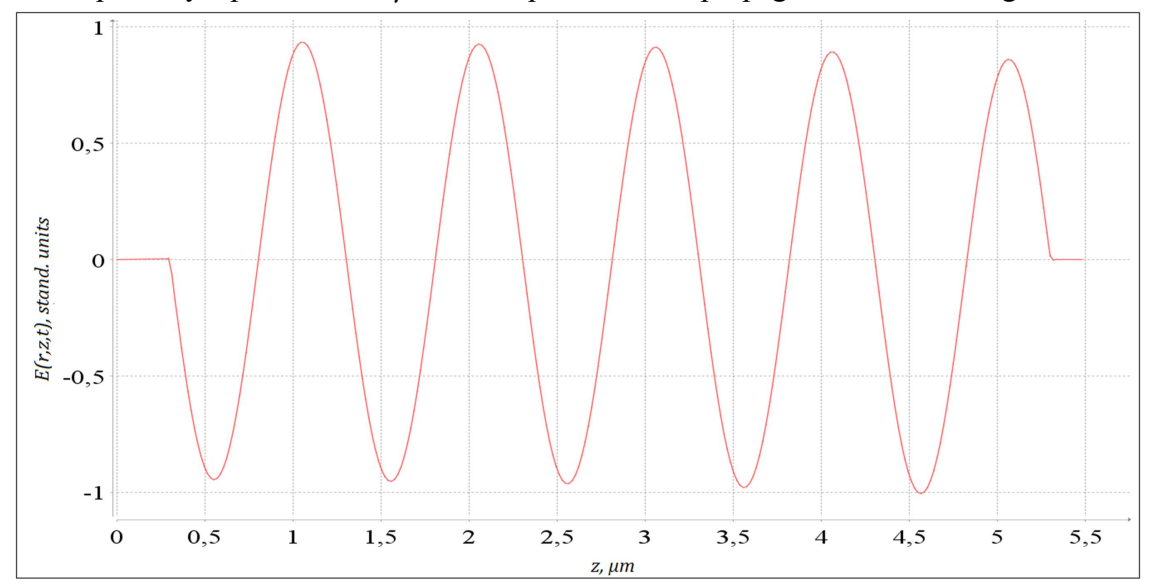

Fig. 1. Modeling the distribution piecewise smooth impulse in wave conductor, separation $r=1 \mu \mathrm{m}$.

For the case of smooth pulse described by function $\psi_{2}(r, t)$ if $\omega^{*}=\frac{\omega}{10}$ and, $\varphi(r)=J_{0}\left(\lambda_{1} r\right)$ solution of boundary-value problem is as follows:

$$
\begin{aligned}
& E(r, z, t)=J_{0}\left(\lambda_{1} r\right)\left[\sum_{k=0}^{\infty} \frac{c_{k}}{\omega_{k}} \sin \left(v_{k} z\right)\left(0.5 a_{3}(t)+a_{4}(t)+a_{5}(t)\right)+\sin (\omega t) \sin ^{2}\left(\frac{\omega t}{10}\right)\right], \text { if } t \in\left[0 ; t^{*}\right] \\
& E(r, z, t)=J_{0}\left(\lambda_{1} r\right) \sum_{k=0}^{\infty} \sin \left(v_{k} z\right)\left(a_{6}\left(t^{*}\right) \cos \left(\omega_{k}\left(t-t^{*}\right)\right)+\frac{a_{7}\left(t^{*}\right)}{\omega_{k}} \sin \left(\omega_{k}\left(t-t^{*}\right)\right)\right), \text { if } t \in\left(t^{*} ; T\right] .
\end{aligned}
$$

In the last formulas, we used the following notations:

$$
\begin{aligned}
& a_{3}(t)=\frac{\hat{\omega}^{2}-\omega^{2}}{\omega^{2}-\omega_{k}^{2}}\left(\omega \sin \omega_{k} t-\omega_{k} \sin \omega t\right), \\
& a_{4}(t)=5 \frac{0.16 \omega^{2}-0.25 \hat{\omega}^{2}}{16 \omega^{2}-25 \omega_{k}^{2}}\left(4 \omega \sin \omega_{k} t-5 \omega_{k} \sin \frac{4}{5} \omega t\right), a_{5}(t)=5 \frac{0.36 \omega^{2}-0.25 \hat{\omega}^{2}}{36 \omega^{2}-25 \omega_{k}^{2}}\left(6 \omega \sin \omega_{k} t-5 \omega_{k} \sin \frac{6}{5} \omega t\right), \\
& a_{6}(t)=\frac{c_{k}}{\omega_{k}}\left(0.5 a_{3}\left(t^{*}\right)+a_{4}\left(t^{*}\right)+a_{5}\left(t^{*}\right)\right)+c_{k} \sin (\omega t) \sin ^{2}\left(\frac{\omega t}{10}\right), \\
& a_{7}(t)=\frac{c_{k}}{\omega_{k}}\left(0.5 a_{3}^{\prime}\left(t^{*}\right)+a_{4}^{\prime}\left(t^{*}\right)+a_{5}^{\prime}\left(t^{*}\right)\right)+c_{k}\left(\sin (\omega t) \sin ^{2}\left(\frac{\omega t}{10}\right)^{\prime}\right),
\end{aligned}
$$

$\mu_{1}$ is a root of an equation $J_{0}(\mu R)=0$.

The process of propagating a piecewise-smooth pulse has shown in figure 2 . 


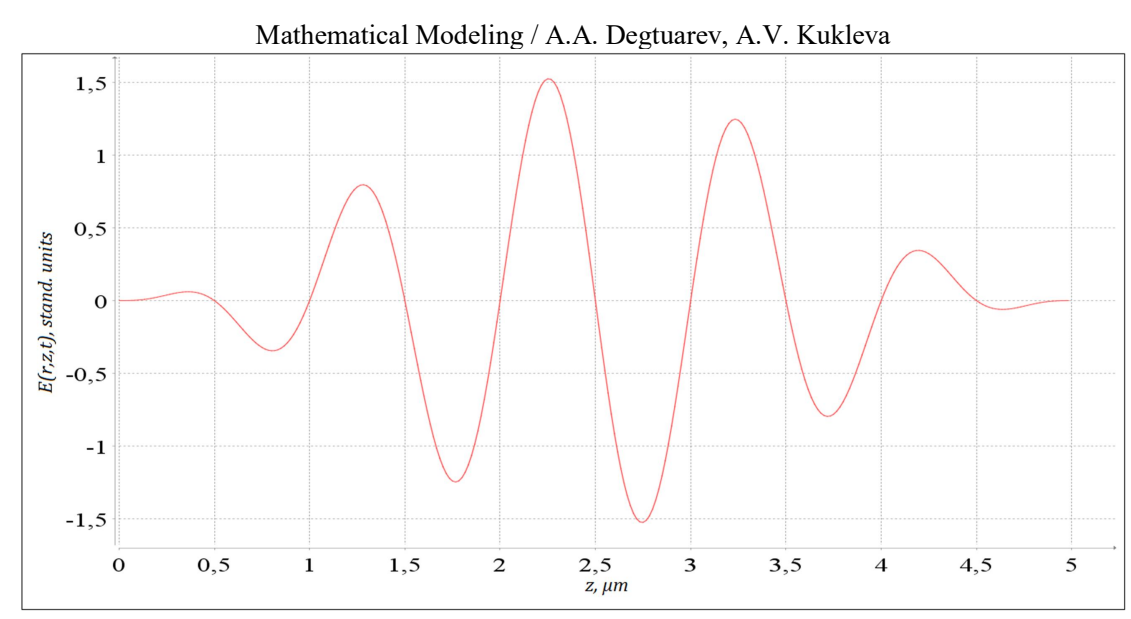

Fig.2. Modeling of smooth pulse in wave conductor, separation $r=1 \mu m$.

\section{Series truncation error control}

A computer program simulating the spread of pulse truncation of the infinite series implied above.

If we can get an estimate a balance number of $E(r, z, t)=\sum_{k=1}^{N} u_{k}(r, z, t)$ in the form of

$$
\left|R_{N}\right|=\left|\sum_{k=N+1}^{\infty} u_{k}(r, z, t)\right| \leq \Phi(N),
$$

where $\Phi(N)$ is the positive monotonically decreasing function if $N \rightarrow+\infty$, this assessment can be used to control the truncation error. To do this, we need only find $N(\varepsilon)$, is the least value $N$, satisfy the inequality $\Phi(N) \leq \varepsilon$, and for approximate calculation of function values $E(x, z, t)$ use a partial amount $E_{N(\varepsilon)}=\sum_{n=1}^{N(\varepsilon)} e_{n}(x, z, t)$.

In this case, the actual error of the calculated value of a function $E$ at the selected point does not exceed the required level $\varepsilon$, that is

$$
\varepsilon_{\text {fact }}=\left|E-E_{N(\varepsilon)}\right|=\left|R_{N(\varepsilon)}\right| \leq \Phi(N(\varepsilon)) \leq \varepsilon .
$$

For the above two ways to specify the light pulse residues had been received by the relevant rows with the following $t^{*}$ and $w^{*}:$

$$
w^{*}=\frac{\pi c}{5 \lambda}, t^{*}=\frac{10 \lambda}{c} .
$$

In the case of piecewise smooth impulse, that described function $\psi_{1}(r, t)$, assessed takes the following form:

$$
\left|E_{N}(r, z, t)\right| \leq \frac{8 L n}{\pi(2 N+1)}\left(\frac{1.003}{\lambda}+\frac{2 n L\left(\omega^{2}-\hat{\omega}^{2}\right)}{\pi^{2} c^{2}\left(3+2 N-\frac{4 n L}{\lambda}\right)}\right),
$$

as for the case of smooth pulse, that described function $\psi_{2}(r, t)$, assessed takes the following form:

$$
\left|E_{N}(r, z, t)\right| \leq \frac{0.16 n^{2} L^{2} \omega^{2}}{c^{2} \pi^{3}(2 N+1)^{2}} .
$$

It should be noted that recorded higher truncation error estimates infinite series are uniform for all independent variables.

\section{The method of refinement of the number of summable elements of a series using a computational experiment}

Proposed evaluation are not ideal because they are using strict inequalities, and also they are uniform for all independent variables. That is why using of estimates results in adding more elements than is necessary to achieve the required accuracy. In this case, it is advisable to apply a technique, which reduces the degree of redundancy terms in the partial sum, and in so doing guarantees the achievement of required accuracy [3].

Let $N$ positive integer, satisfies the inequality $N \leq N\left(\varepsilon_{1}\right)$, where $\varepsilon_{1}<\varepsilon$, number $N\left(\varepsilon_{1}\right)$ found by the rule described in paragraph 4. Then for partial amount $E_{N}$ the actual error will satisfy the inequality: $\varepsilon_{\text {fact }}(N)=\left|E-E_{N}\right| \leq\left|E-E_{N\left(\varepsilon_{1}\right)}\right|+\left|E_{N\left(\varepsilon_{1}\right)}-E_{N}\right|$ 
Changing $N$ within the boundaries $N\left(\varepsilon_{1}\right) \geq N \geq 1$, find lowest value $N\left(\varepsilon_{2}\right)$, when running the inequality $\left|E_{N\left(\varepsilon_{1}\right)}-E_{N}\right| \leq \varepsilon_{2}$, where $\varepsilon_{2}=\varepsilon-\varepsilon_{1}$.

For this choice $\varepsilon_{2}$ and equity of the previous inequality, the actual error $\varepsilon_{\text {fact }}\left(N\left(\varepsilon_{2}\right)\right)$ do not exceed value $\varepsilon$.

Thus, to reduce the number of summands in the partial amount, we must:

1) Specify the number of $\varepsilon_{1}<\varepsilon$ and then find the value $N\left(\varepsilon_{1}\right)$, that the smallest value $N$, satisfy the inequality $\Phi(N) \leq \varepsilon_{1}$.

2) Changing a variable $N$ from the value $N\left(\varepsilon_{1}\right)$ downward, find the smallest of its value that satisfies the inequality $\left|E_{N\left(\varepsilon_{1}\right)}-E_{N}\right| \leq \varepsilon_{2}$. The resulting value is $N\left(\varepsilon_{2}\right)$.

3) Changing value with sample spacing $\varepsilon_{1}$ and $\varepsilon_{2}$ so, to $\varepsilon_{1}+\varepsilon_{2}=\varepsilon$, run the steps 1) and 2) again.

4) Of all the values $N\left(\varepsilon_{2}\right)$, obtained in step 3), select the smallest.

As a result of the use of this algorithm, it can be expected that the number of summable elements $N\left(\varepsilon_{2}\right)$ in the partial sum will be reduced significantly as compared with the number of $N(\varepsilon)$ while maintaining safeguards for accuracy, i.e. $\varepsilon_{\text {fact }}\left(N\left(\varepsilon_{2}\right)\right) \leq \varepsilon$. In tables 1 and 2 are the results of computational experiments, aimed at reducing the number of summands in partial amounts. The calculations have been carried out with the following parameters:

$\lambda=1 \mu \mathrm{m}, n=1, L=7 \mu \mathrm{m}, R=5 \mu \mathrm{m}, c=3 \cdot 10^{14} \mu \mathrm{m} / \mathrm{s}, r=1 \mu \mathrm{m}, z=1 \mu \mathrm{m}, t=\frac{t c}{n} \mu \mathrm{m}$.

Asked value $\varepsilon$ in increments of the maximum value of the amplitude of the wave.

Table 1. The dependence of the summands number $N(\varepsilon)$ and $N\left(\varepsilon_{2}\right)$ of coordinate $t$

with different values $\varepsilon$ for piecewise smooth impulse.

\begin{tabular}{rrrrrr}
$\varepsilon$ & $10^{-1}$ & $10^{-2}$ & $10^{-3}$ & $10^{-4}$ & $10^{-5}$ \\
\hline$N(\varepsilon)$ & 131 & 1019 & 9844 & 98079 & 980434 \\
\hline$t, \mu m$ & \multicolumn{5}{c}{$N\left(\varepsilon_{2}\right)$} \\
\hline 0.9 & 13 & 48 & 231 & 3116 & 9906 \\
\hline 0.999 & 37 & 306 & 1241 & 6774 & 26632 \\
\hline 0.99999 & 37 & 312 & 3072 & 35599 & 126836 \\
\hline 1 & 37 & 312 & 3075 & 37713 & 377122 \\
\hline 1.00001 & 37 & 312 & 3072 & 35599 & 126836 \\
\hline$\varepsilon$ & $10^{-1}$ & $10^{-2}$ & $10^{-3}$ & $10^{-4}$ & $10^{-5}$ \\
\hline 1.001 & 37 & 306 & 1241 & 6774 & 26633 \\
\hline 1.1 & 16 & 68 & 320 & 3119 & 9906 \\
\hline 1.7 & 19 & 34 & 124 & 1286 & 4086 \\
\hline 2.5 & 15 & 32 & 96 & 928 & 984 \\
\hline 4 & 13 & 25 & 66 & 612 & 643 \\
\hline 5.1 & 16 & 30 & 75 & 649 & 1436 \\
\hline 5.9 & 17 & 28 & 324 & 3116 & 3258 \\
\hline 5.999 & 47 & 355 & 1262 & 6422 & 9906 \\
\hline 5.99999 & 47 & 466 & 4672 & 35599 & 26632 \\
\hline 6 & 47 & 467 & 4672 & 37713 & 126836 \\
\hline 6.00001 & 47 & 465 & 4671 & 35599 & 377122 \\
\hline 6.001 & 47 & 383 & 1461 & 6423 & 126836 \\
\hline 6.1 & 17 & 30 & 360 & 3119 & 26634
\end{tabular}

Table 2. The dependence of the summands number $N(\varepsilon)$ and $N\left(\varepsilon_{2}\right)$ of coordinate $t$ with different values $\varepsilon$ for smooth pulse.

\begin{tabular}{rrrrrr}
$\varepsilon$ & $10^{-2}$ & $10^{-3}$ & $10^{-4}$ & $10^{-5}$ & $10^{-6}$ \\
\hline$N(\varepsilon)$ & 21 & 36 & 113 & 357 & 1128 \\
\hline$t, \mu m$ & \multicolumn{5}{c}{$N\left(\varepsilon_{2}\right)$} \\
\hline 0.9 & 15 & 18 & 28 & 62 & 132 \\
\hline 0.999 & 15 & 18 & 28 & 61 & 136 \\
\hline 0.99999 & 14 & 17 & 28 & 62 & 126 \\
\hline 1 & 15 & 18 & 27 & 67 & 141 \\
\hline 1.000001 & 10 & 21 & 37 & 91 & 186 \\
\hline 1.001 & 10 & 22 & 42 & 101 & 211 \\
\hline 1.1 & 15 & 17 & 33 & 61 & 132 \\
\hline 1.7 & 10 & 17 & 37 & 81 & 181 \\
\hline 2.5 & 13 & 15 & 26 & 67 & 146 \\
\hline 4 & 15 & 22 & 46 & 101 & 216
\end{tabular}


From the table it can be seen that the number of summands, using uniform assessments for the respective series truncation allows you to get only the rough partial sums of lengths. These values are repeatedly exceed the values obtained from the application of the above algorithm. As can be seen from table 1, to calculate the tension of the electric field in the foreground and background areas of wave fronts requires a much larger number of terms, for example, in the range $1.7 \mu \mathrm{m} \leq t \leq 5.1 \mu \mathrm{m}$ order enough 4086 parts to achieve precision $10^{-5}$, while in the range $0.9 \mu m \leq t \leq 1.1 \mu m$ we want 377122 parts. This increase in the number of summands is a consequence of the weak function breaks $\psi_{1}(r, t)$, significantly slowing down the convergence of series. For the case of smooth pulse, function description $\psi_{2}(r, t)$ the uneven distribution of values $N\left(\varepsilon_{2}\right)$ for different $t$ turns out to be negligible.

\section{Conclusion}

Developed and implemented programmatically algorithm provides adjustment of the partial sums length of infinite series, obtained in the course of solving boundary value problem for the wave equation. For practical application of the algorithm, it is of fundamental importance to first obtain an upper estimate for the remainder of the Fourier series that determines the solution of the boundary value problem.

The application of developed algorithm for specific series that describe the distribution of momentum in circular waveguide section allowed multiple times (from 3 up to 1500 times and more for Piecewise-smooth momentum and from 2 to 5 times for the case of smooth pulse) to reduce length of the partial sums of the series.

\section{References}

[1] Feng X. A high-order compact scheme for the one-dimensional Helmholtz equation with a discontinuous coefficient. International Journal of Computer Mathematics 2012; 1: 1-7.

[2] Degtyarev AA, Kozlova ES. Investigation of accuracy of numerical solution of the one-way helmholtz equation by method of computational experiment. Computer Optics 2012; 36(1): 36-45.

[3] Degtyarev AA, Praslova MO. Estimation of the error in the solution of the wave equation in the problem of modeling the propagation of a light pulse in a planar waveguide. Proceedings of the International Conference Information Technoloy and Nanotechnology. Samara, Samara National Research University, 2016; 852-859.

[4] Kozlova ES, Kotlyar VV. Simulation of ultrafast 2d light pulse. Computer Optics 2012; 36(2): $158-164$.

[5] Kotlyar VV, Kozlova ES. Simmulations of sommerfeld and brillouin precursors in the medium with frequency dispersion using numerical method of solving wave equations. Computer Optics 2013; 37(2): 146-154.

[6] Fuchs U, Zeitner U, Tunnermann A. Ultra-short pulse propagation in complex optical system. Optics Express 2005; 13(10): 3852-3861.

[7] Tikhonov AN, Samarskiy A.A. Equations of mathematical physics. M.: Nauka, 1972; 736 p. 